\title{
Relationships between Hippocampal Atrophy, White Matter Disruption, and Gray Matter Hypometabolism in Alzheimer's Disease
}

\author{
Nicolas Villain, ${ }^{1}$ Béatrice Desgranges, ${ }^{1}$ Fausto Viader, ${ }^{1,2}$ Vincent de la Sayette, ${ }^{1,2}$ Florence Mézenge, ${ }^{1}$ Brigitte Landeau, ${ }^{1}$ \\ Jean-Claude Baron, ${ }^{3}$ Francis Eustache, ${ }^{1}$ and Gaël Chételat ${ }^{1}$ \\ ${ }^{1}$ Institut National de la Santé et de la Recherche Médicale-Ecole Pratique des Hautes Etudes-Université de Caen/Basse-Normandie, Unité U923, \\ Groupement d'Intérêt Public Cyceron, Centre Hospitalier Universitaire (CHU) Côte de Nacre, 14074 Caen, France, ${ }^{2}$ Département de Neurologie, CHU Côte \\ de Nacre, 14033 Caen Cedex, France, and ${ }^{3}$ Department of Clinical Neurosciences, Neurology Unit, University of Cambridge, Cambridge CB2 2SP, United \\ Kingdom
}

In early Alzheimer's disease (AD), the hippocampal region is the area most severely affected by cellular and structural alterations, yet glucose hypometabolism predominates in the posterior association cortex and posterior cingulate gyrus. One prevalent hypothesis to account for this discrepancy is that posterior cingulate hypometabolism results from disconnection from the hippocampus through disruption of the cingulum bundle. However, only partial and indirect evidence currently supports this hypothesis. Thus, using structural magnetic resonance imaging and 2-[ $\left[{ }^{18} \mathrm{~F}\right]$ fluoro-2-deoxy-D-glucose positron emission tomography in 18 patients with early AD, we assessed the relationships between hippocampal atrophy, white matter integrity, and gray matter metabolism by means of a whole-brain voxel-based correlative approach. We found that hippocampal atrophy is specifically related to cingulum bundle disruption, which is in turn highly correlated to hypometabolism of the posterior cingulate cortex but also of the middle cingulate gyrus, thalamus, mammillary bodies, parahippocampal gyrus, and hippocampus (all part of Papez's circuit), as well as the right temporoparietal associative cortex. These results provide the first direct evidence supporting the disconnection hypothesis as a major factor contributing to the early posterior hypometabolism in $\mathrm{AD}$. Disruption of the cingulum bundle also appears to relate to hypometabolism in a large connected network over and above the posterior cingulate cortex, encompassing the whole memory circuit of Papez (consistent with the key location of this white matter tract within this loop) and also, but indirectly, the right posterior association cortex.

Key words: Alzheimer's disease; hippocampus; white matter; deafferentation; morphometry; PET; positron emission tomography

\section{Introduction}

Prototypical brain glucose metabolism alterations in patients with Alzheimer's disease (AD), also observed in patients with amnestic mild cognitive impairment (MCI), are characterized by early involvement of the posterior cingulate cortex (PCC), subsequently spreading to the neighboring precuneus and temporoparietal neocortical areas (Chételat et al., 2003a; Nestor et al., 2004; Mevel et al., 2007).

There is a striking discrepancy between this hypometabolic profile and the well described pattern of gray matter (GM) atrophy mainly affecting first the medial and then the lateral temporal areas,

\footnotetext{
Received Jan. 31, 2008; accepted April 30, 2008.

This work was supported by Institut National de la Santé et de la Recherche Médicale (INSERM), including the INSERM MD-PhD Program, Programme Hospitalier de Recherche Clinique (Ministère de la Santé), Région BasseNormandie, and Association France Alzheimer. We thank M. Fouquet and K. Mevel for their helpful comments regarding this work; C. Lalevée and A. Pélerin for help with neuropsychological assessments; B. Dupuy and D. Hannequin for their contribution to the recruitment of patients; M. H. Noël, M. C. Onfroy, D. Luet, O. Tirel, and L. Barré for help with neuroimaging data acquisition; and the people who participated in this study.

Correspondence should be addressed to Dr. Gaël Chételat, Institut National de la Santé et de la Recherche Médicale-Ecole Pratique des Hautes Etudes-Université de Caen/Basse-Normandie, Unité U923, Groupement d'Intérêt Public Cyceron, Boulevard H. Becquerel, BP 5229, 14074 Caen Cedex, France. E-mail: chetelat@cyceron.fr. DOI:10.1523/JNEUROSCI.1392-08.2008

Copyright $\odot 2008$ Society for Neuroscience $\quad 0270-6474 / 08 / 286174-08 \$ 15.00 / 0$
}

before extending to the cingulate cortex and temporoparietal regions (Matsuda et al., 2002; Chételat et al., 2003b; Thompson et al., 2003; Whitwell et al., 2007), consistent with the course of neurofibrillary degenerations in AD (Braak and Braak, 1991). The most robust and widely held hypothesis to account for this discrepancy is that the hypometabolism of the posterior association cortex and more particularly the PCC does not only result from local neuropathological processes, but mostly reflects the distant effect of neuronal damage in the hippocampal formation (including the hippocampus proper and the parahippocampal cortex), i.e., a disconnection process (Jobst et al., 1992; Meguro et al., 2001; Matsuda et al., 2002; Smith, 2002; Chételat et al., 2003b; Nestor et al., 2003, 2004).

There is long-standing evidence for this "diaschisis" hypothesis from neuropathological studies. Hyman et al. (1984) first pointed out the specific alteration of hippocampal and parahippocampal neurons involved in connecting the hippocampal formation with other brain structures. Subsequently, Pearson et al. (1985) hypothesized a mechanism of neuropathological alterations expansion in $\mathrm{AD}$ along the projecting fibers, notably hippocampal projection neurons. And Jobst et al. (1992) first postulated a distant effect of the hippocampal region atrophy to explain the reduced blood flow in the posterior areas. 
Table 1. Demographic characteristics of subjects

\begin{tabular}{lll}
\hline & Healthy aged subjects & Patients with Alzheimer's disease \\
\hline $\begin{array}{l}\text { Number } \\
\text { Males/females }\end{array}$ & 15 & 18 \\
Age (years) & $7 / 8$ & $3 / 15$ \\
$\quad$ Mean \pm SD & $66.5 \pm 7.3$ & \\
$\quad$ Range & $60-84$ & $69.5 \pm 5.2$ \\
MMS & & $60-82$ \\
$\quad$ Mean \pm SD & & $24.3 \pm 2.6$ \\
Range & & $20-29$ \\
\hline
\end{tabular}

This diaschisis hypothesis has since been repeatedly mentioned, and further supported by indirect or partial evidence. For instance, PCC hypometabolism was observed in nonhuman primates after hippocampal and parahippocampal lesions (Meguro et al., 1999; Machado et al., 2008). In addition, significant positive correlations were reported in patients with $\mathrm{AD}$ between hippocampal formation size on the one hand, and either PCC activation during a memory task (Garrido et al., 2002; Remy et al., 2005) or posterior associative cortical areas resting-state metabolism (Meguro et al., 2001). Recent studies using diffusion tensor imaging (DTI) in $\mathrm{AD}$ have documented disruption of the cingulum bundle (Rose et al., 2000; Fellgiebel et al., 2005; Teipel et al., 2007; Zhang et al., 2007), a white matter (WM) tract linking the hippocampus proper and parahippocampal cortex to the PCC (Mufson and Pandya, 1984; Morris et al., 1999; Mori et al., 2005; Schmahmann and Pandya, 2006), as well as its relationship with global and hippocampal atrophy (Xie et al., 2005; Firbank et al., 2007).

Although the above findings argue in favor of a disconnection hypothesis to explain PCC hypometabolism in early AD, there is to date no direct evidence that PCC hypometabolism results from hippocampal formation atrophy via cingulum bundle disruption. The aim of the present study is to further explore this hypothesis by assessing the relationships between hippocampal atrophy, WM integrity, and GM metabolism in patients with clinically probable AD.

\section{Materials and Methods \\ Subjects}

Eighteen patients were studied, all right-handed with at least 8 years of education. At the time of the study, none of the patients was being or had been treated with specific medication, such as antiacetylcholinesterasic drugs. All were prospectively selected using standard National Institute of Neurological and Communicative Disorders and Stroke-Alzheimer's Disease and Related Disorders Association diagnostic criteria for probable AD (McKhann et al., 1984), based on an extensive neuropsychological examination as detailed previously (Desgranges et al., 1998; Baron et al., 2001; Chételat et al., 2008). Only patients who had both T1-weighted magnetic resonance imaging (MRI) and positron emission tomography (PET) coupled to 2- $\left[{ }^{18} \mathrm{~F}\right]$ fluoro-2-deoxy-D-glucose $\left({ }^{18} \mathrm{FDG}\right)$ examinations and mini-mental state $>20$ (i.e., mild $\mathrm{AD}$ ) were included in the present study. Fifteen unmedicated healthy controls who also underwent both MRI and PET were also studied, all right-handed with at least 8 years of education. They were screened for the absence of cerebrovascular risk factors, mental disorder, substance abuse, head trauma, and significant MRI or biological abnormality. The two groups were matched for age, and although women were overrepresented in the $\mathrm{AD}$ sample compared with controls, this difference was not significant $\left(\chi^{2}\right.$ Yates correction $\left.=2.21 ; p=0.13\right)$ (for demographic data, see Table 1). The subjects were the same as those studied by Chételat et al. (2008). All the subjects were fully cooperative and free from behavioral disturbances. They all gave their consent to the study after detailed information was provided to them, and the PET procedure was approved by the Ethical Committee of the University of Caen. The study was done in line with the Declaration of Helsinki.

\section{Imaging data acquisition}

For each subject, a high-resolution T1-MRI scan was obtained, which consisted in a set of 128 adjacent axial slices parallel to the anterior commissure-posterior commissure line with slice thickness $1.5 \mathrm{~mm}$ and pixel size $0.9375 \times 0.9375 \mathrm{~mm}^{2}$ using the spoiled gradient echo sequence [repetition time, $10.3 \mathrm{~ms}$; echo time, $2.1 \mathrm{~ms}$; field of view (FoV), $240 \times$ $180 \mathrm{~mm}^{2}$; matrix, $256 \times 192$ ]. All the MRI data sets were acquired on the same scanner (1.5 T Signa Advantage echospeed; General Electric).

Each subject also underwent a PET study within days of the MRI study. Data were collected using the Siemens ECAT Exact HR+ PET device with isotropic resolution of $4.2 \times 4.2 \times 4.6 \mathrm{~mm}^{3}$ (axial FoV, $158 \mathrm{~mm}$ ). The patients had been fasting for at least $4 \mathrm{~h}$ before scanning. To minimize anxiety, the PET procedure was explained in detail beforehand. The head was positioned on a head rest according to the canthomeatal line and gently restrained with straps. ${ }^{18} \mathrm{FDG}$ uptake was measured in the resting condition, with eyes closed, in a quiet and dark environment. A catheter was introduced in a vein of the arm to inject the radiotracer. After ${ }^{68} \mathrm{Ga}$ transmission scans, $3-5 \mathrm{mCi}$ of ${ }^{18} \mathrm{FDG}$ was injected as a bolus at time 0 , and a $10 \mathrm{~min}$ PET data acquisition started at $50 \mathrm{~min}$ postinjection period. Sixty-three planes were acquired with septa out (three-dimensional acquisition), using a voxel size of $2.2 \times 2.2 \times 2.43 \mathrm{~mm}^{3}(x y z)$.

\section{Imaging data handling and transformation}

The procedure used for data handling and transformation was based on the study by Chételat et al. (2008), but WM MRI data were also used for the purpose of the present study and specific GM and WM masks were created. The statistical analyses performed here (see below) were also strictly different.

MRI data. The MRI data sets were analyzed using Statistical Parametric Mapping (SPM2; http://www.fil.ion.ucl.ac.uk/spm) and the optimized voxel-based morphometry (VBM) procedure described in detail previously (Good et al., 2001) and already used in our laboratory (Chételat et al., 2002, 2005). Briefly, the procedure included the creation of customized templates of the whole brain and the GM, WM, and CSF sets using the MRI data from the whole combined patient and control samples $(n=33)$. The original MRI data sets were then segmented into SPM (implying a reversible affine normalization step) using these customized templates as priors. The resultant original (i.e., in native space) GM data sets were then spatially normalized onto the GM customized priors, respectively, to determine the optimal normalization parameters, which were then applied to the corresponding original whole-brain MRI scans. Finally, the "optimally" normalized whole-brain MRI data sets were segmented into SPM, and the resultant GM and WM partitions were smoothed [14.6 mm full width at half-maximum (FWHM)] (see below) and masked (see below).

PET data. The PET data were first corrected for partial volume effect (PVE) attributable to both CSF and WM using the optimal voxel-by-voxel method originally proposed by Müller-Gärtner et al. (1992), and slightly modified as proposed by Rousset et al. (1998). This method, referred to as "modified Müller-Gärtner," is described in detail by Quarantelli et al. (2004), and has already been applied in our laboratory (Chételat et al., 2003a, 2008; Mevel et al., 2007). All image processing steps for PVE correction were performed using the "PVE-lab" software (Quarantelli et al., 2004). Using SPM2, PVE-corrected PET data sets were then coregistered onto their respective MRI and spatially normalized onto the same GM customized template as that used for the spatial normalization of MRI data, by reapplying the normalization parameters estimated from the VBM procedure. The normalized PET data sets were then smoothed ( $14 \mathrm{~mm}$ FWHM) (see below). The resulting PET images were divided by their individual vermis FDG uptake value to control for individual variations in global PET measures, following the procedure already used in our laboratory (for details, see Mevel et al., 2007). Smoothed and scaled PET data were then masked using the same GM mask as that used for the GM partition obtained from MRI data (see below).

Differential smoothing. To blur individual variations in gyral anatomy and increase the signal-to-noise ratio, the spatially normalized GM and WM partitions and the corrected and spatially normalized PET data sets were smoothed. We used the standard Gaussian kernel of $14 \mathrm{~mm}$ FWHM for the PET data. Because PET and MRI data had different original spatial resolutions, differential smoothing was applied to obtain images of 
equivalent effective smoothness, and thus of identical resultant resolution (Richardson et al., 1997; Van Laere and Dierckx, 2001). To this end, we used a Gaussian kernel of $14.6 \mathrm{~mm}$ FWHM for the MRI GM and WM data, resulting in an effective smoothness identical to PET images smoothed at $14 \mathrm{~mm}$ FWHM (Poline et al., 1995).

Masking. The GM, WM, and PET images obtained following the steps above were masked so as to include only GM or WM voxels of interest and to prevent any overlap between voxels included in analyses with GM and those with WM. The GM mask was obtained by first thresholding the GM customized template above a value of 0.3 , corresponding to a $>30 \%$ chance for the voxel to belong to GM. The resultant "permissive" GM mask was then manually adjusted to exclude any voxel of WM (such as the interfaces between CSF and WM misclassified as GM: fornix, ventricles edges,... ). A WM mask was then obtained by first creating a "permissive" WM mask by thresholding the WM customized template above a value of 0.15 , excluding the pons and cerebellum and then excluding all voxels included in the final GM mask. The same binary GM mask was applied to both the GM and the PET data sets and binary WM mask was applied to the WM data set.

Z-score maps. The smoothed and masked GM, $\mathrm{WM}$, and PET images were used to create $Z$-score maps [(patient individual value - controls mean)/controls SD], for each patient and each modality. The GM, WM, and PET $Z$-score maps thus obtained for each patient were then entered in the correlative analyses described below.

\section{Statistical analysis}

WM atrophy. Because the profiles of GM atrophy and hypometabolism have been described previously (Chételat et al., 2008), only the pattern of WM atrophy will be detailed here. Group differences were assessed to obtain maps of statistically significant WM atrophy in AD patients relative to controls, using the smoothed and masked WM data set obtained as described above and the two-sample $t$ test SPM2 routine.

Correlation between atrophied hippocampal formation GM Z-score and whole-brain WM $\mathrm{Z}$-score maps. The full procedure of data analysis is summarized in Figure 1. First, mean hippocampal formation GM $Z$-scores were extracted from the significantly atrophied hippocampal formation GM voxels (obtained by comparing $\mathrm{AD}$ patients with controls using the two-sample $t$ test SPM2 routine), using a $p$ [false discovery rate (FDR)-corrected for multiple comparisons] $<0.05$ threshold with $k>$ 20 voxels (see Fig. $1 B$ ). Because both the parahippocampal gyrus and the hippocampus proper send direct projections via the cingulum bundle (and thus potentially liable to cingulum atrophy), we used the mean GM $Z$-score (termed "hippocampal formation GM $Z$-score") across the significantly atrophied voxels of these two anatomical labels when assessing correlations with WM $Z$-score maps. Anatomical labeling was based on the Anatomical Automatic Labeling (AAL) software (Tzourio-Mazoyer et al., 2002) after normalization of the anatomically labeled Montreal Neurological Institute (MNI) template onto our customized template.
A

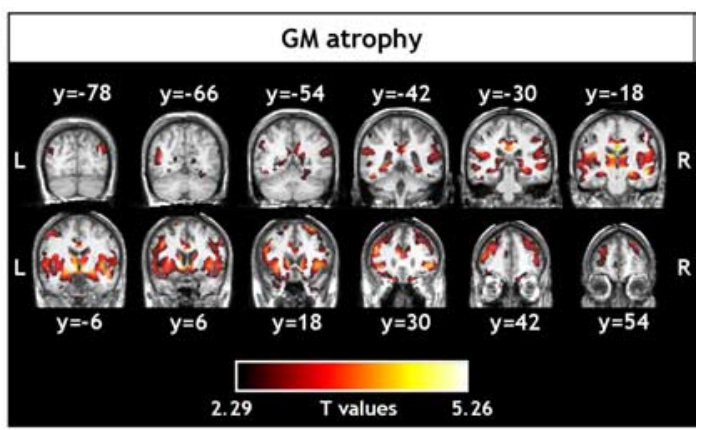

C

Correlation between hippocampal

formation GM Z-scores and whole brain WM Z-score maps
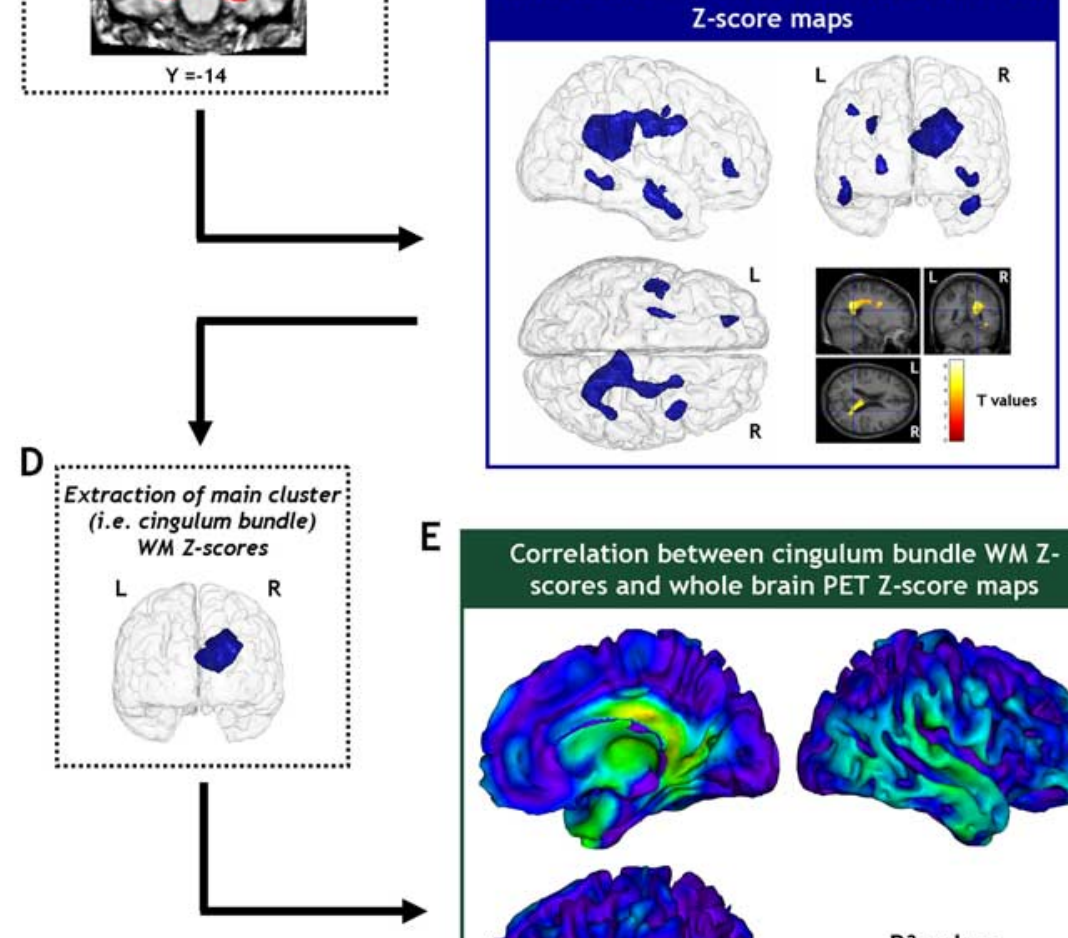

E

Correlation between cingulum bundle WM Zscores and whole brain PET Z-score maps

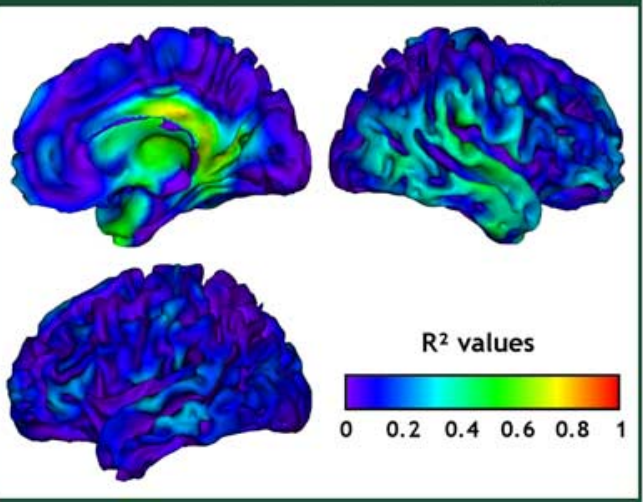

Figure 1. Illustration of the different analysis steps and their corresponding findings (thresholded results are displayed at $p<0.05$, FDR-corrected, with $k>20$ voxels). First, from the voxels of significant GM atrophy in $\mathrm{AD}$ compared with controls $(\boldsymbol{A})$, the mean GM Z-score of the bilateral hippocampal formation was extracted for each subject $(\boldsymbol{B})$ and entered as covariate in a correlative analysis with whole-brain WM Z-score maps. The results of this correlative analysis are illustrated $(\boldsymbol{C})$ using the Anatomist $3 D$ render (www.brainvisa.info; superior, right, and posterior views) as well as the SPM cross-sectional render centered at 22, $-48,20(x, y, z ; M N I$ coordinates). The WM mean Z-score in the main cluster of the previous analysis (i.e., the caudal part of the cingulum) was then extracted for each subject ( $\boldsymbol{D}$ ) to be entered as covariate in a correlative analysis with the whole-brain PET $Z$-score maps. The results of this analysis are displayed as $R^{2}$ maps onto three-dimensional surface renderings $(\boldsymbol{E})$ (www. brainvisa.info). L, Left; $R$, right.

Second, positive correlations were computed across the $18 \mathrm{AD}$ patients between hippocampal formation GM $Z$-scores and whole-brain WM $Z$-score maps, using the "single-subject: covariates only" SPM2 routine. This procedure allows the performance of linear regressions between one or more variables of interest and each voxel of the maps entered into the analysis. Note that we also compared the correlations with GM $Z$-scores in the hippocampus proper to that in the parahippocampal gyrus to assess whether these two structures show any significant difference in their relationships with WM atrophy.

Correlation between WM Z-scores and whole-brain PETZ-score maps. The 


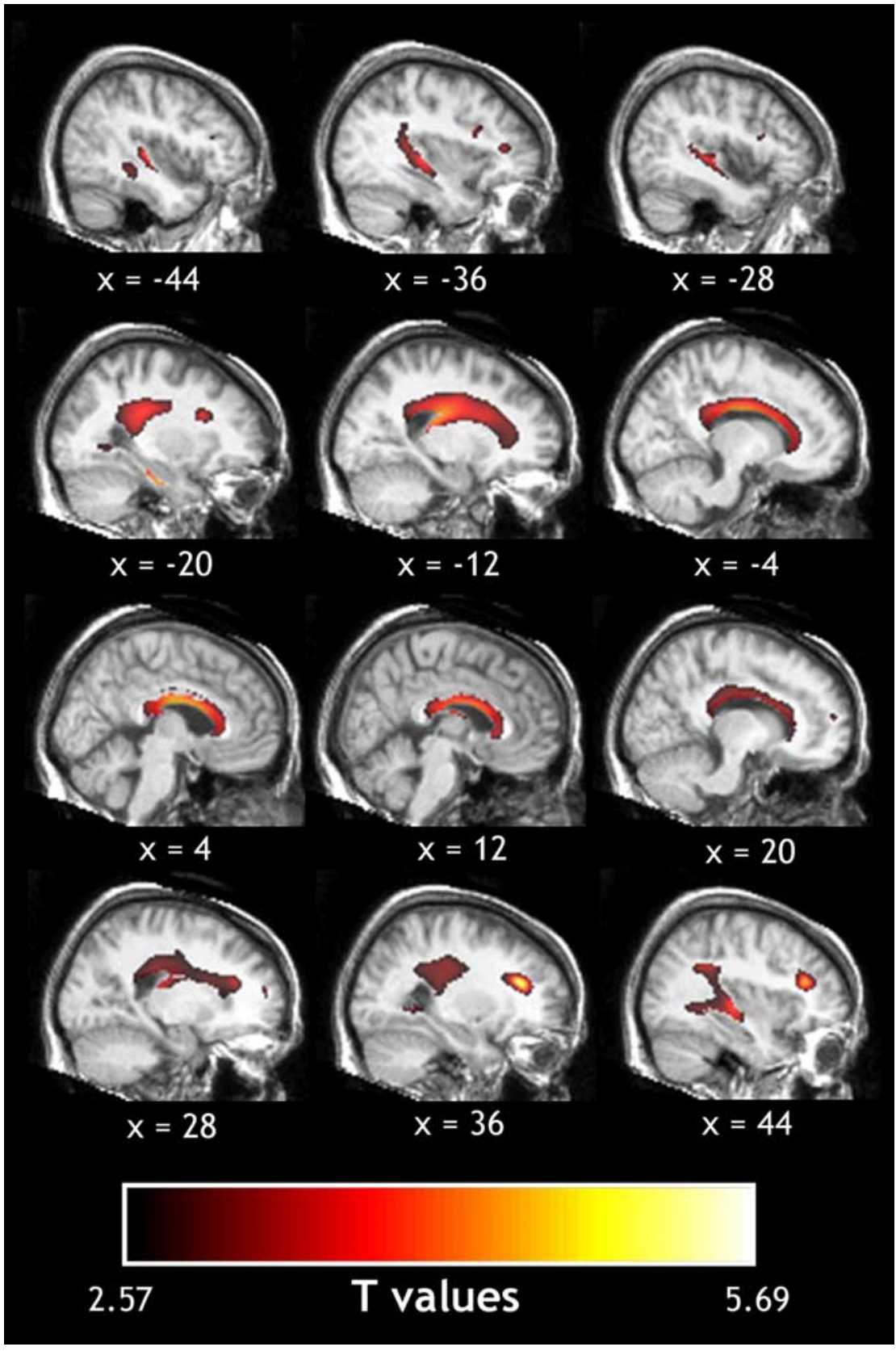

Figure 2. Areas of significant decrease in WM density in patients with Alzheimer's disease compared with controls ( $p<$ 0.05 , FDR-corrected, with $k>20$ voxels) as projected onto sagittal sections (MNI coordinates).

mean WM $Z$-score from the main cluster of the previous correlation analysis (between hippocampal formation GM $Z$-scores and WM $Z$-score maps) thresholded at FDR-corrected $p<0.05$ with $k>20$ voxels, was extracted for each patient (see Fig. $1 D$ ). These individual values of WM $Z$-score were then entered as covariates in a statistical analysis of positive correlations with the PET Z-score maps, using the "single-subject: covariates only" SPM2 routine. The resulting SPM-T map was then converted to a $R^{2}$ map.

Statistical threshold and display of results. SPM-T maps of all previously described analyses were thresholded using a $p$ (FDR-corrected for multiple comparisons) $<0.05$ threshold with a $k>20$ voxels. To avoid type II errors attributable to overconservative threshold, results were also displayed using a more liberal uncorrected $p<0.05$ threshold with a $k>$ 20 voxels. Correlations were only assessed in the physiologically expected (i.e., positive) direction, corresponding to the hypothesis of a relationship between GM atrophy, WM atrophy, and GM hypometabolism (see Introduction).

Anatomical localization was based on the superimposition of the SPM-T and $R^{2}$ maps onto the customized template, and identification of the localization using the AAL software and anatomical atlases (Talairach and Tournoux, 1988; Tzourio-Mazoyer et al., 2002; Mori et al., 2005). The findings were also rendered using the publicly available "Anatomist/BrainVISA" software (www.brainvisa.info).

\section{Results}

The profiles of GM atrophy and hypometabolism, described in detail by Chételat et al. (2008), were consistent with the characteristic pattern of brain alterations in $\mathrm{AD}$, mainly involving the hippocampal formation and temporal neocortex for the former (for an overview of GM atrophy, see Fig. 1A), and posterior associative areas including the PCC for the latter.

\section{WM atrophy}

Regions of significant WM atrophy are displayed in Figure 2. They included the corpus callosum (CC), fornix, cingulate WM including the cingulum bundle (involving both its rostral and caudal portions), parahippocampal clusters corresponding to the perforant path, and temporal WM clusters (Table 2).

\section{Correlation analysis}

Positive correlations between hippocampal formation GM $Z$-scores and WM $Z$-score maps are displayed in Figure 1C. Significant correlations were found with the right cingulate WM including the caudal part of the cingulum bundle, the largest and most significant cluster, but also with frontal and parahippocampal WM clusters mainly corresponding to the rostral and most caudal parts of the cingulum bundle, respectively (Table 2, Fig. 1C). When lowering the statistical threshold to $p<0.05$ uncorrected, the correlation concerned both the left and right cingulum bundles along their whole length, curving around the temporal horns of the lateral ventricles and joining together all the previously described clusters from the frontal to the parahippocampal fibers (Fig. 3). The posterior part of the CC (splenium) was also found significantly correlated using this more permissive threshold (Fig. 3).

No significant difference [ $p$ (FDR-corrected for multiple comparisons $)<0.05$ ] was found in the correlations between the WM $Z$-scores maps and the GM $Z$-scores for the hippocampus proper and the parahippocampal gyrus (data not shown).

Positive correlations between the mean WM $Z$-scores of the right cingulate WM cluster (centered at 27-53 31, MNI coordinates) (Table 2), corresponding to the main cluster of the previous analysis (FDR-corrected $p<0.05$ ) (Table 2), and PET $Z$-score maps were found in the middle cingulate cortex, the PCC (mostly its retrosplenial part; BA29/30) and precuneus, hippocampal and parahippocampal regions, as well as several subcortical structures including the thalamus and the mammillary bodies (for whole-brain $R^{2}$ results, see Table 2, Fig. $1 E$; and for the SPM-T map thresholded at FDR-corrected $p<0.05$, see Fig. 
4). The $R^{2}$ maps revealed $R^{2}$ values between 0.5 and 0.8 within these clusters (Fig. $1 E$ ). A few tiny right temporoparietal clusters were also found (Table 2), with high $R^{2}$ values $(0.4-0.5)$ in the inferior parietal lobule as well as superior, middle, and inferior temporal gyri (Fig. $1 E$ ). Adding age and mini-mental state (MMS) score as covariates showed essentially unchanged partial regressions (data not shown). Note that the same findings were obtained when the cingulum cluster WM $Z$-scores were derived from a $p<0.05$ (uncorrected) threshold, leading to inclusion of the whole bilateral cingulum bundle fibers (data not shown).

\section{Discussion}

The present study revealed a pattern of WM atrophy mainly involving the CC, fornix, cingulum, perforant path, and temporal WM. There were strong positive correlations between hippocampal formation GM $Z$-score and WM $Z$-scores of the cingulum bundle including both its caudal (between the hippocampal formation and the PCC) and rostral (between the PCC and the frontal cortex) parts, and, to a lesser degree, WM $Z$-score of the splenium. Finally, the cingulum WM $Z$-score was itself strongly related to PET $Z$-scores in the posterior and middle cingulate gyri, thalamus, mammillary bodies, parahippocampal cortex, hippocampus, and right temporoparietal cortex.

The profile of WM atrophy observed in $\mathrm{AD}$ in the present study using VBM and showing a major involvement of the cingulum bundle, is highly consistent with the disconnection hypothesis. The involvement of this tract as well as the CC, fornix, perforant path, and temporal WM is consistent with previous findings from DTI studies that reported diffusivity increases or anisotropy decreases in the same set of WM areas (Fellgiebel et al., 2004; Medina et al., 2006; Xie et al., 2006; Huang et al., 2007; Teipel et al., 2007; Zhang et al., 2007). Atrophy of the CC and perforant path have also already been highlighted in previous VBM studies of AD (Chaim et al., 2007) and MCI (Stoub et al., 2006), respectively. That highly consistent patterns of WM atrophy are defined using two highly different methods of acquisition and analysis such as DTI and VBM is noteworthy, and our study further emphasizes the validity and sensitivity of automatic whole-brain $\mathrm{VBM}$ to assess WM atrophy in $\mathrm{AD}$.

One first main objective with this study was to assess the relationships between hippocampal formation GM atrophy and whole-brain WM atrophy. We found a strong and specific correlation of hippocampal formation atrophy with cingulum bundle atrophy, documenting that disruption of the cingulum bundle is specifically related to atrophy of the hippocampal formation. Our findings are consistent with a previous report using DTI that showed a significant correlation between cingulum bundle fractional anisotropy and hippocampal volume (Xie et al., 2005). We also found a weaker correlation with the splenium, likely corresponding to the tapetum, an interhemispheric tract located at the posterior-most part of the CC that links both temporal lobes together (Mori et al., 2005; Schmahmann and Pandya, 2006). These results thus suggest that hippocampal formation atrophy may be related to disruption of the connections between the medial temporal lobe and the controlateral temporal cortex.

Moreover, these relationships were found to be relatively specific. Because both the hippocampal formation and the cingulum bundle were markedly atrophied, this correlation could reflect association rather than causality (i.e., it could be attributable to an independent effect of AD on these two structures). However, correlations were not found to involve any atrophied WM tract (even when lowering the statistical threshold), but only those known to be connected to the hippocampal formation (Figs. 1C, 4). Furthermore, there also seems to be some specificity among the hippocampal formation tracts, because for instance the fornix, which is both atrophied and connected to the hippocampus (Duvernoy and Vannson, 1998), did not exhibit a significant correlation. This suggests the involvement of alternative causes for WM tract alteration over and above hippocampal damage, as well as particular vulnerability in AD pathology for those hippocampal neurons that project through the cingulum bundle.

The strongest correlation with hippocampal formation atrophy concerned the caudal part of the cingulum bundle, which mainly includes fibers connecting the parahippocampal gyrus and hippocampus proper to the PCC (Mufson and Pandya, 1984; Kobayashi and Amaral, 2003, 2007; Mori et al., 2005; Schmahmann and Pandya, 2006). Correlations were also found for the 

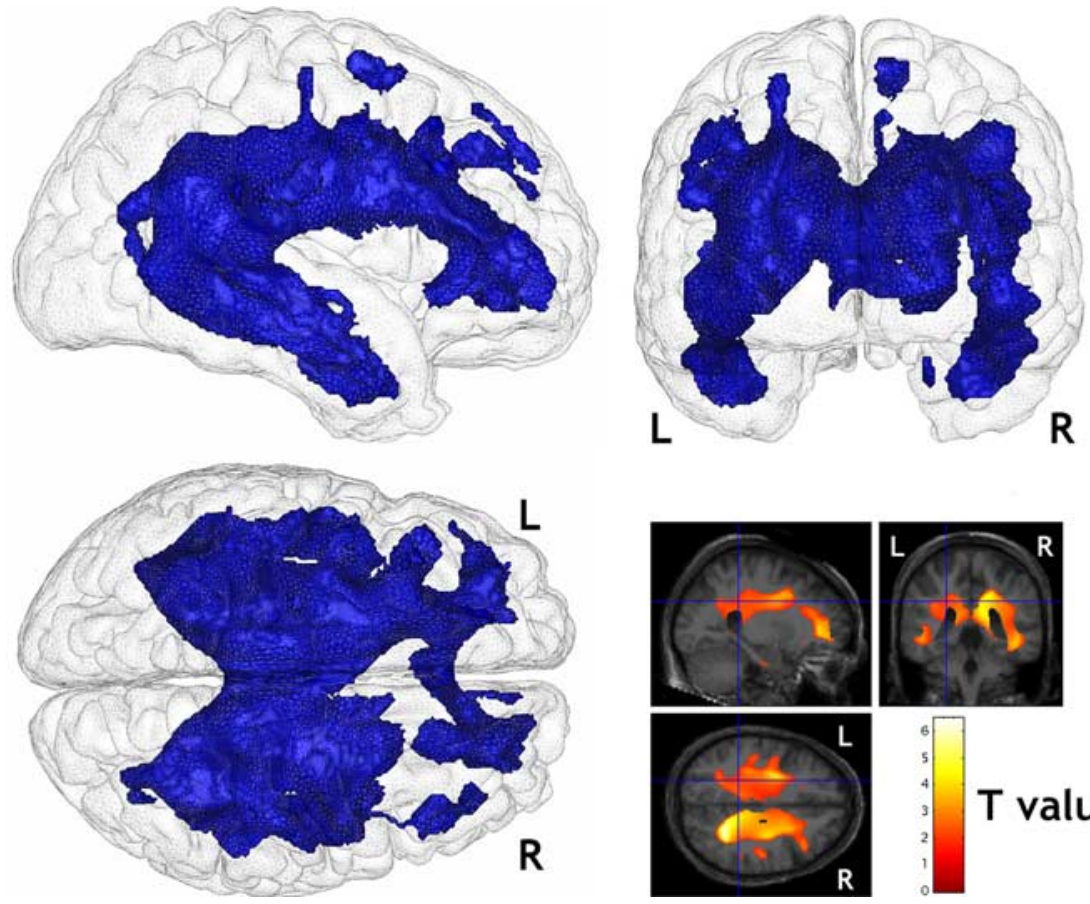

Figure 3. Significant correlations between the GM Z-score of the hippocampal formation and the whole-brain WM Z-score maps, thresholded at $p<0.05$ uncorrected $(k>20)$ and displayed on Anatomist 3D render (www.brainvisa.info; superior, right, and posterior views) as well as onto SPM cross-sectional render centered at $-24,-40,30(x, y, z$; MNI coordinates). L, Left; $R$, right.

rostral part of the cingulum bundle, which notably comprises hippocampo-frontal (Mufson and Pandya, 1984; Morris et al., 1999) and retrosplenial-frontal tracts. It suggests that hippocampal formation atrophy in $\mathrm{AD}$ also leads to the disruption of fronto-hippocampal connections, not only directly, but also indirectly through the damaged retrosplenial PCC (BA29 and 30), because this area "provides the most direct and most massive route for information flow between the dorsolateral frontal cortex and the medial temporal lobe" (Kobayashi and Amaral, 2003) (see also Morris et al., 1999; Kobayashi and Amaral, 2007). These hypotheses are further supported by functional connectivity studies showing a decrease in fronto-hippocampal connectivity at rest (Wang et al., 2006; Allen et al., 2007) as well as during a memory task (Grady et al., 2001) in AD.

Our second main objective was to assess the relationships between cingulum bundle atrophy and cortical hypometabolism. The results highlighted strong correlation with posterior cingulate hypometabolism and more specifically its retrosplenial part (Figs. $1 E, 4$ ). This is the first direct evidence relating PCC hypometabolism to hippocampal atrophy via the cingulum bundle disruption, further supporting the "diaschisis" hypothesis for PCC hypometabolism in AD. The precise localization of this correlation to the retrosplenium is entirely consistent with the neuroanatomy, because BA29 and 30 are the PCC subregions mainly connected to the hippocampal formation via the cingulum bundle (Kobayashi and Amaral, 2003, 2007). The $R^{2}$ values for this correlation reached $0.5-0.8$, i.e., cingulum bundle atrophy explained $\sim 50-80 \%$ of PCC metabolism variance independently of age and MMS score. Thus, neither age nor disease severity, but the loss of hippocampal inputs itself, accounts for the majority of PCC hypometabolism in AD. This is consistent with the fact that the hippocampal-cingulate fibers represent the main input to the retrosplenium (Kobayashi and Amaral, 2003).

\section{$T$ values}

Cingulum bundle atrophy was also found to be related to hypometabolism of the mammillary bodies, thalamus, middle cingulate gyrus, parahippocampal gyrus, and hippocampus. This finding might reflect the key location of the cingulum bundle within the hippocampo-mammillothalamo-cingulo-parahippocampal loop, i.e., the Papez circuit (Papez, 1937), because it includes connections between the cingulate gyrus and both the thalamus and the parahippocampal gyrus (Mufson and Pandya, 1984). Thus, cingulum bundle atrophy likely disrupts Papez's circuit, leading to hypometabolism affecting its nodes.

Relatively high $R^{2}$ values $(0.4-0.5)$ also involved the right temporoparietal cortex. This finding is somewhat unexpected because the temporal neocortex does not receive direct projections from the cingulum bundle (Mufson and Pandya, 1984; Mori et al., 2005; Schmahmann and Pandya, 2006). However, this correlation is weaker than that for the above-mentioned regions directly connected via the cingulum, and as such may reflect an indirect relationship. Using a correlative approach in humans, Vogt et al. (2006) recently highlighted regions monosynaptically or multisynaptically linked to the ventral PCC, pointing to a large association temporoparietal region that encompasses the area found here. Thus, cingulum bundle might induce temporoparietal hypometabolism through an indirect connection involving the ventral PCC. It is noteworthy that this correlation was found with the right temporoparietal cortex only. Evidence of asymmetric pathological mechanisms in $\mathrm{AD}$ have been frequently documented, notably concerning GM atrophy (Thompson et al., 1998, 2003) and hypometabolism (Haxby and Rapoport, 1986). Recently, several studies characterizing the earliest metabolic alterations in $\mathrm{AD}$ pointed to the right temporoparietal areas (Chételat et al., 2003a; Hirao et al., 2005; Ishii et al., 2005; Kawachi et al., 2006). Although the lateralization obtained here remains to be elucidated, it might reflect a still unexplained asymmetric mechanism of brain alteration in AD.

Strikingly, we found no significant correlation with the metabolism of other structures known to be connected through the cingulum bundle such as frontal areas. This may be attributable to the fact that, whereas hippocampal fibers represent the major input to the retrosplenium, hippocampal inputs to other cortical regions and particularly to anterior frontal areas are much less prominent. Consequently, the influence of cingulum bundle disruption would be expected to be weaker in these anterior regions. This lack of correlation might also in part result from functional compensations, particularly in the prefrontal cortex where such processes have been repeatedly reported in $\mathrm{AD}$ (Grady et al., 2003; Remy et al., 2005).

The use of $Z$-scores in the correlation analyses in this study was preferred here to account for the multimodality analysis, but could have induced a bias because by definition $Z$-scores depend on $\mathrm{SD}$, which could vary across imaging modalities. However, we repeated the same analyses using GM/WM density and PET maps instead of $Z$-score maps and similar results were obtained (data not shown). Another issue relates to anatomical accuracy limitation inherent to voxel-based method implying normalization and 


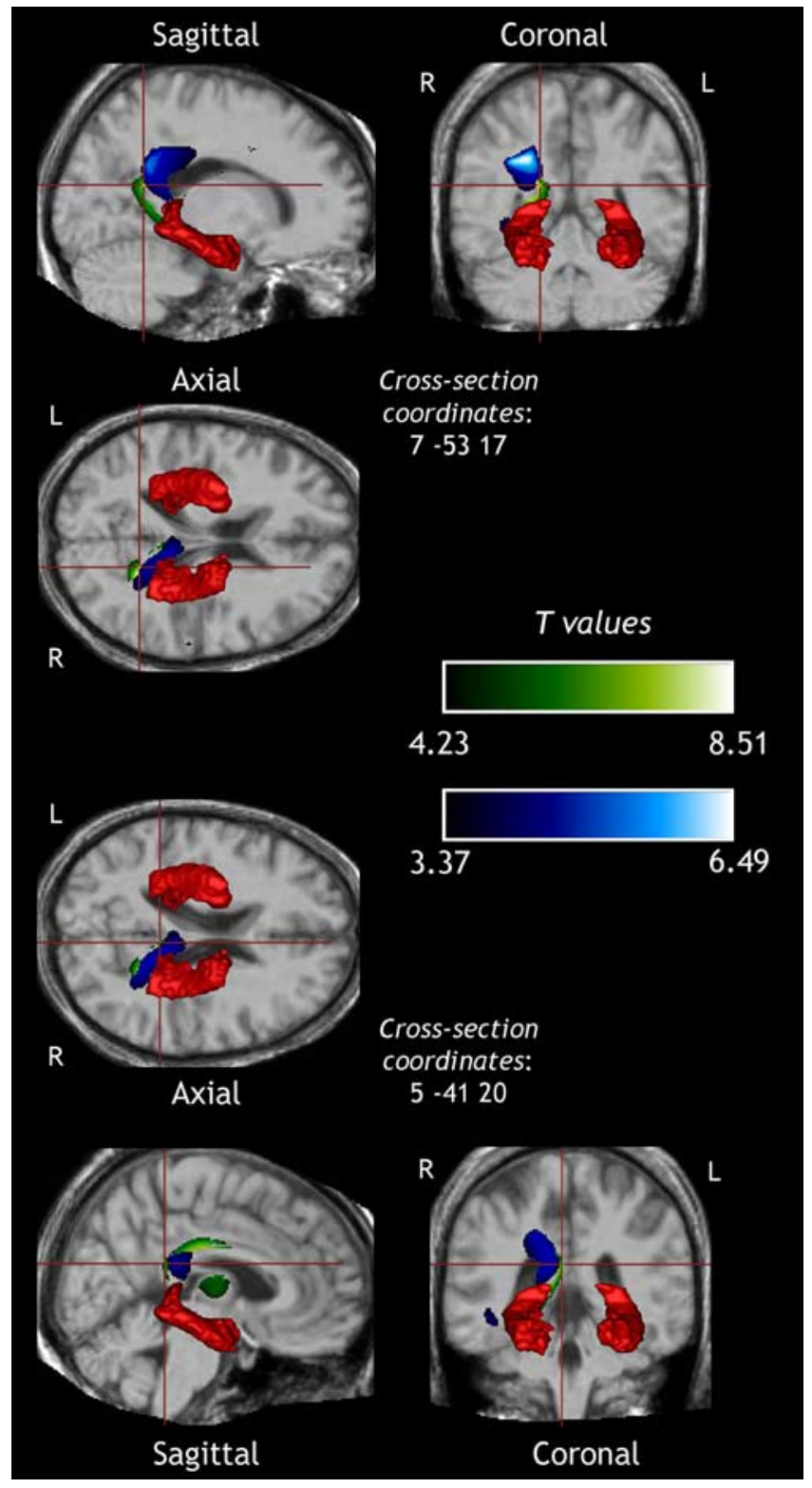

Figure 4. Overall display of the main findings from this study as projected onto the same brain views. The significantly atrophied hippocampal formation voxels used to extract mean GM Z-scores for the correlation with WM Z-score maps are represented in red. The cingulum cluster resulting from the previous correlative analysis and used to extract the mean WM Z-scores subsequently correlated to PET Z-score maps, is shown in blue ( $p<0.05$, FDR-corrected, $k>$ 20 voxels). Finally, statistically significant positive correlations between WM Z-scores of the cingulum cluster, extracted from the first correlation, and PET Z-score maps are illustrated in green ( $p<0.05$, FDR-corrected, with $k>20$ voxels). L, Left; $R$, right.

smoothing. Consequently, SPM findings can be smeared in space (e.g., the cingulate WM clusters obtained here exceed the expected single-subject cingulum bundle width, so that we cannot exclude the involvement of adjacent noncingulum WM). Moreover, results in small brain structures such as the fornix and mammillary bodies should be considered with some caution given this methodological limitation.

To sum up, by relating the PCC hypometabolism in AD to hippocampal formation atrophy through disruption of the cingulum bundle, this study provides strong and direct support to diaschisis as the link between these two characteristic alterations of AD. Furthermore, posterior cingulate hypometabolism in $\mathrm{AD}$ may result from an early disturbance of fronto-hippocampal communications, and cingulum bundle disruption plays a major role in the dysfunction of the major connection nodes of Papez's circuit.

\section{References}

Allen G, Barnard H, McColl R, Hester AL, Fields JA, Weiner MF, Ringe WK, Lipton AM, Brooker M, McDonald E, Rubin CD, Cullum CM (2007) Reduced hippocampal functional connectivity in Alzheimer disease. Arch Neurol 64:1482-1487.

Baron JC, Chételat G, Desgranges B, Perchey G, Landeau B, de la Sayette V, Eustache F (2001) In vivo mapping of gray matter loss with voxel-based morphometry in mild Alzheimer's disease. NeuroImage 14:298-309.

Braak H, Braak E (1991) Neuropathological stageing of Alzheimer-related changes. Acta Neuropathol (Berl) 82:239-259.

Chaim TM, Duran FL, Uchida RR, Perico CA, de Castro CC, Busatto GF (2007) Volumetric reduction of the corpus callosum in Alzheimer's disease in vivo as assessed with voxel-based morphometry. Psychiatry Res 154:59-68.

Chételat G, Desgranges B, de la Sayette V, Viader F, Eustache F, Baron JC (2002) Mapping gray matter loss with voxel-based morphometry in mild cognitive impairment. NeuroReport 13:1939-1943.

Chételat G, Desgranges B, de la Sayette V, Viader F, Eustache F, Baron JC (2003a) Mild cognitive impairment: Can FDG-PET predict who is to rapidly convert to Alzheimer's disease? Neurology 60:1374-1377.

Chételat G, Desgranges B, de la Sayette V, Viader F, Berkouk K, Landeau B, Lalevee C, Le Doze F, Dupuy B, Hannequin D, Baron JC, Eustache F (2003b) Dissociating atrophy and hypometabolism impact on episodic memory in mild cognitive impairment. Brain 126:1955-1967.

Chételat G, Landeau B, Eustache F, Mezenge F, Viader F, de la Sayette V, Desgranges B, Baron JC (2005) Using voxel-based morphometry to map the structural changes associated with rapid conversion in MCI: a longitudinal MRI study. NeuroImage 27:934-946.

Chételat G, Desgranges B, Landeau B, Mezenge F, Poline JB, de la Sayette V, Viader F, Eustache F, Baron JC (2008) Direct voxel-based comparison between grey matter hypometabolism and atrophy in Alzheimer's disease. Brain 131:60-71.

Desgranges B, Baron JC, de la Sayette V, Petit-Taboue MC, Benali K, Landeau B, Lechevalier B, Eustache F (1998) The neural substrates of memory systems impairment in Alzheimer's disease. A PET study of resting brain glucose utilization. Brain 121:611-631.

Duvernoy HM, Vannson JL (1998) The human hippocampus. New York: Springer.

Fellgiebel A, Wille P, Muller MJ, Winterer G, Scheurich A, Vucurevic G, Schmidt LG, Stoeter P (2004) Ultrastructural hippocampal and white matter alterations in mild cognitive impairment: a diffusion tensor imaging study. Dement Geriatr Cogn Disord 18:101-108.

Fellgiebel A, Muller MJ, Wille P, Dellani PR, Scheurich A, Schmidt LG, Stoeter P (2005) Color-coded diffusion-tensor-imaging of posterior cingulate fiber tracts in mild cognitive impairment. Neurobiol Aging 26:1193-1198.

Firbank MJ, Blamire AM, Krishnan MS, Teodorczuk A, English P, Gholkar A, Harrison R, O'Brien JT (2007) Atrophy is associated with posterior cingulate white matter disruption in dementia with Lewy bodies and Alzheimer's disease. NeuroImage 36:1-7.

Garrido GE, Furuie SS, Buchpiguel CA, Bottino CM, Almeida OP, Cid CG, Camargo CH, Castro CC, Glabus MF, Busatto GF (2002) Relation between medial temporal atrophy and functional brain activity during memory processing in Alzheimer's disease: a combined MRI and SPECT study. J Neurol Neurosurg Psychiatry 73:508-516.

Good CD, Johnsrude IS, Ashburner J, Henson RN, Friston KJ, Frackowiak RS (2001) A voxel-based morphometric study of ageing in 465 normal adult human brains. NeuroImage 14:21-36.

Grady CL, Furey ML, Pietrini P, Horwitz B, Rapoport SI (2001) Altered brain functional connectivity and impaired short-term memory in Alzheimer's disease. Brain 124:739-756.

Grady CL, McIntosh AR, Beig S, Keightley ML, Burian H, Black SE (2003) Evidence from functional neuroimaging of a compensatory prefrontal network in Alzheimer's disease. J Neurosci 23:986-993.

Haxby JV, Rapoport SI (1986) Abnormalities of regional brain metabolism in Alzheimer's disease and their relation to functional impairment. Prog Neuropsychopharmacol Biol Psychiatry 10:427-438.

Hirao K, Ohnishi T, Hirata Y, Yamashita F, Mori T, Moriguchi Y, Matsuda H, Nemoto K, Imabayashi E, Yamada M, Iwamoto T, Arima K, Asada T 
(2005) The prediction of rapid conversion to Alzheimer's disease in mild cognitive impairment using regional cerebral blood flow SPECT. NeuroImage 28:1014-1021.

Huang J, Friedland RP, Auchus AP (2007) Diffusion tensor imaging of normal-appearing white matter in mild cognitive impairment and early Alzheimer disease: preliminary evidence of axonal degeneration in the temporal lobe. AJNR Am J Neuroradiol 28:1943-1948.

Hyman BT, Van Hoesen GW, Damasio AR, Barnes CL (1984) Alzheimer's disease: cell-specific pathology isolates the hippocampal formation. Science 225:1168-1170.

Ishii K, Sasaki H, Kono AK, Miyamoto N, Fukuda T, Mori E (2005) Comparison of gray matter and metabolic reduction in mild Alzheimer's disease using FDG-PET and voxel-based morphometric MR studies. Eur J Nucl Med Mol Imaging 32:959-963.

Jobst KA, Smith AD, Barker CS, Wear A, King EM, Smith A, Anslow PA, Molyneux AJ, Shepstone BJ, Soper N (1992) Association of atrophy of the medial temporal lobe with reduced blood flow in the posterior parietotemporal cortex in patients with a clinical and pathological diagnosis of Alzheimer's disease. J Neurol Neurosurg Psychiatry 55:190-194.

Kawachi T, Ishii K, Sakamoto S, Sasaki M, Mori T, Yamashita F, Matsuda H, Mori E (2006) Comparison of the diagnostic performance of FDG-PET and VBM-MRI in very mild Alzheimer's disease. Eur J Nucl Med Mol Imaging 33:801-809.

Kobayashi Y, Amaral DG (2003) Macaque monkey retrosplenial cortex: II. Cortical afferents. J Comp Neurol 466:48-79.

Kobayashi Y, Amaral DG (2007) Macaque monkey retrosplenial cortex: III. Cortical efferents. J Comp Neurol 502:810-833.

Machado CJ, Snyder AZ, Cherry SR, Lavenex P, Amaral DG (2008) Effects of neonatal amygdala or hippocampus lesions on resting brain metabolism in the macaque monkey: a microPET imaging study. NeuroImage 39:832-846.

Matsuda H, Kitayama N, Ohnishi T, Asada T, Nakano S, Sakamoto S, Imabayashi E, Katoh A (2002) Longitudinal evaluation of both morphologic and functional changes in the same individuals with Alzheimer's disease. J Nucl Med 43:304-311.

McKhann G, Drachman D, Folstein M, Katzman R, Price D, Stadlan EM (1984) Clinical diagnosis of Alzheimer's disease: report of the NINCDSADRDA Work Group under the auspices of Department of Health and Human Services Task Force on Alzheimer's Disease. Neurology 34:939-944.

Medina D, Toledo-Morrell L, Urresta F, Gabrieli JD, Moseley M, Fleischman D, Bennett DA, Leurgans S, Turner DA, Stebbins GT (2006) White matter changes in mild cognitive impairment and $\mathrm{AD}$ : a diffusion tensor imaging study. Neurobiol Aging 27:663-672.

Meguro K, Blaizot X, Kondoh Y, Le Mestric C, Baron JC, Chavoix C (1999) Neocortical and hippocampal glucose hypometabolism following neurotoxic lesions of the entorhinal and perirhinal cortices in the non-human primate as shown by PET. Implications for Alzheimer's disease. Brain 122:1519-1531.

Meguro K, Le Mestric C, Landeau B, Desgranges B, Eustache F, Baron JC (2001) Relations between hypometabolism in the posterior association neocortex and hippocampal atrophy in Alzheimer's disease: a PET/MRI correlative study. J Neurol Neurosurg Psychiatry 71:315-321.

Mevel K, Desgranges B, Baron JC, Landeau B, de la Sayette V, Viader F, Eustache F, Chételat G (2007) Detecting hippocampal hypometabolism in Mild Cognitive Impairment using automatic voxel-based approaches. NeuroImage 37:18-25.

Mori S, Wakana S, Nagae-Poetscher LM, van Zilj PCM (2005) MRI atlas of human white matter. Amsterdam: Elsevier.

Morris R, Pandya DN, Petrides M (1999) Fiber system linking the middorsolateral frontal cortex with the retrosplenial/presubicular region in the rhesus monkey. J Comp Neurol 407:183-192.

Mufson EJ, Pandya DN (1984) Some observations on the course and composition of the cingulum bundle in the rhesus monkey. J Comp Neurol 225:31-43.

Müller-Gärtner HW, Links JM, Prince JL, Bryan RN, McVeigh E, Leal JP, Davatzikos C, Frost JJ (1992) Measurement of radiotracer concentration in brain gray matter using positron emission tomography: MRI-based correction for partial volume effects. J Cereb Blood Flow Metab 12:571-583.

Nestor PJ, Fryer TD, Ikeda M, Hodges JR (2003) Retrosplenial cortex (BA 29/30) hypometabolism in mild cognitive impairment (prodromal Alzheimer's disease). Eur J Neurosci 18:2663-2667.

Nestor PJ, Scheltens P, Hodges JR (2004) Advances in the early detection of Alzheimer's disease. Nat Rev Neurosci Suppl S:S34-S41.
Papez JW (1937) A proposed mechanism of emotion. Arch Neurol Psychiatry 38:725-743.

Pearson RC, Esiri MM, Hiorns RW, Wilcock GK, Powell TP (1985) Anatomical correlates of the distribution of the pathological changes in the neocortex in Alzheimer disease. Proc Natl Acad Sci USA 82:4531-4534.

Poline JB, Worsley KJ, Holmes AP, Frackowiak RS, Friston KJ (1995) Estimating smoothness in statistical parametric maps: variability of $p$ values. J Comput Assist Tomogr 19:788-796.

Quarantelli M, Berkouk K, Prinster A, Landeau B, Svarer C, Balkay L, Alfano B, Brunetti A, Baron JC, Salvatore M (2004) Integrated software for the analysis of brain PET/SPECT studies with partial-volume-effect correction. J Nucl Med 45:192-201.

Remy F, Mirrashed F, Campbell B, Richter W (2005) Verbal episodic memory impairment in Alzheimer's disease: a combined structural and functional MRI study. NeuroImage 25:253-266.

Richardson MP, Friston KJ, Sisodiya SM, Koepp MJ, Ashburner J, Free SL, Brooks DJ, Duncan JS (1997) Cortical grey matter and benzodiazepine receptors in malformations of cortical development. A voxel-based comparison of structural and functional imaging data. Brain 120:1961-1973.

Rose SE, Chen F, Chalk JB, Zelaya FO, Strugnell WE, Benson M, Semple J, Doddrell DM (2000) Loss of connectivity in Alzheimer's disease: an evaluation of white matter tract integrity with colour coded MR diffusion tensor imaging. J Neurol Neurosurg Psychiatry 69:528-530.

Rousset OG, Ma Y, Evans AC (1998) Correction for partial volume effects in PET: principle and validation. J Nucl Med 39:904-911.

Schmahmann JD, Pandya DN (2006) Fiber pathways of the brain. New York: Oxford UP.

Smith AD (2002) Imaging the progression of Alzheimer pathology through the brain. Proc Natl Acad Sci USA 99:4135-4137.

Stoub TR, Toledo-Morrell L, Stebbins GT, Leurgans S, Bennett DA, Shah RC (2006) Hippocampal disconnection contributes to memory dysfunction in individuals at risk for Alzheimer's disease. Proc Natl Acad Sci USA 103:10041-10045.

Talairach J, Tournoux P (1988) Co-planar stereotaxic atlas of the human brain. New York: Thieme.

Teipel SJ, Stahl R, Dietrich O, Schoenberg SO, Perneczky R, Bokde AL, Reiser MF, Moller HJ, Hampel H (2007) Multivariate network analysis of fiber tract integrity in Alzheimer's disease. NeuroImage 34:985-995.

Thompson PM, Moussai J, Zohoori S, Goldkorn A, Khan AA, Mega MS, Small GW, Cummings JL, Toga AW (1998) Cortical variability and asymmetry in normal aging and Alzheimer's disease. Cereb Cortex 8:492-509.

Thompson PM, Hayashi KM, de Zubicaray G, Janke AL, Rose SE, Semple J, Herman D, Hong MS, Dittmer SS, Doddrell DM, Toga AW (2003) Dynamics of gray matter loss in Alzheimer's disease. J Neurosci 23:994-1005.

Tzourio-Mazoyer N, Landeau B, Papathanassiou D, Crivello F, Etard O, Delcroix N, Mazoyer B, Joliot M (2002) Automated anatomical labeling of activations in SPM using a macroscopic anatomical parcellation of the MNI MRI single-subject brain. NeuroImage 15:273-289.

Van Laere KJ, Dierckx RA (2001) Brain perfusion SPECT: age- and sexrelated effects correlated with voxel-based morphometric findings in healthy adults. Radiology 221:810-817.

Vogt BA, Vogt L, Laureys S (2006) Cytology and functionally correlated circuits of human posterior cingulate areas. NeuroImage 29:452-466.

Wang L, Zang Y, He Y, Liang M, Zhang X, Tian L, Wu T, Jiang T, Li K (2006) Changes in hippocampal connectivity in the early stages of Alzheimer's disease: evidence from resting state fMRI. NeuroImage 31:496-504.

Whitwell JL, Przybelski SA, Weigand SD, Knopman DS, Boeve BF, Petersen RC, Jack Jr CR (2007) 3D maps from multiple MRI illustrate changing atrophy patterns as subjects progress from mild cognitive impairment to Alzheimer's disease. Brain 130:1777-1786.

Xie S, Xiao JX, Wang YH, Wu HK, Gong GL, Jiang XX (2005) Evaluation of bilateral cingulum with tractography in patients with Alzheimer's disease. NeuroReport 16:1275-1278.

Xie S, Xiao JX, Gong GL, Zang YF, Wang YH, Wu HK, Jiang XX (2006) Voxel-based detection of white matter abnormalities in mild Alzheimer disease. Neurology 66:1845-1849.

Zhang Y, Schuff N, Jahng GH, Bayne W, Mori S, Schad L, Mueller S, Du AT, Kramer JH, Yaffe K, Chui H, Jagust WJ, Miller BL, Weiner MW (2007) Diffusion tensor imaging of cingulum fibers in mild cognitive impairment and Alzheimer disease. Neurology 68:13-19. 